\title{
1 Improve Protein Solubility and Activity based on Machine
}

\section{Learning Models}

3 Xi Han ${ }^{1}$, Wenbo Ning ${ }^{1}$, Xiaoqiang $\mathrm{Ma}^{2}$, Xiaonan Wang ${ }^{1, *}$, Kang Zhou ${ }^{1,2, *}$

$4{ }^{1}$ Department of Chemical and Biomolecular Engineering, National University of Singapore,

5 Singapore, 117585.

$6 \quad{ }^{2}$ Disruptive \& Sustainable Technologies for Agricultural Precision, Singapore-MIT Alliance

7 for Research and Technology, Singapore, 138602

$8 *$ To whom correspondence should be addressed.

10 Abstract

11 Improving catalytic ability of protein biocatalysts leads to reduction in the production 12 cost of biocatalytic manufacturing process, but the search space of possible pro13 teins/mutants is too large to explore exhaustively through experiments. To some extent, 14 highly soluble recombinant proteins tend to exhibit high activity. Here, we demonstrate 15 that an optimization methodology based on machine learning prediction model can ef16 fectively predict which peptide tags can improve protein solubility quantitatively. 17 Based on the protein sequence information, a support vector machine model we re18 cently developed was used to evaluate protein solubility after randomly mutated tags 19 were added to a target protein. The optimization algorithm guided the tags to evolve 20 towards variants that can result in higher solubility. Moreover, the optimization results 21 were validated successfully by adding the tags designed by our optimization algorithm 22 to a model protein, expressing it in vivo and experimentally quantifying its solubility 
23 and activity. For example, solubility of a tyrosine ammonium lyase was more than dou-

24 bled by adding two tags to its $\mathrm{N}$ - and $\mathrm{C}$-terminus. Its protein activity was also increased nearly 3.5 fold by adding the tags. Additional experiments also supported that the designed tags were effective for improving activity of multiple proteins and are better than previously reported tags. The presented optimization methodology thus provides a valuable tool for understanding the correlation between amino acid sequence and protein solubility and for engineering protein biocatalysts.

Contact: kang.zhou@nus.edu.sg, chewxia@nus.edu.sg

\section{Introduction}

The exploration of expressing recombinant proteins started in 1976, when human peptide hormone Somatostatin was produced in Escherichia coli ${ }^{1}$. As the most commonly used expression host, E. coli was investigated intensively to improve the expression and activity of recombinant proteins ${ }^{2,3,4}$. Various experimental strategies, such as using protein fusion partners, co-expressing chaperones, choosing suitable promoters, opti-

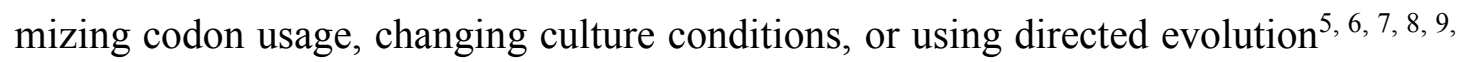
${ }^{10}$, were used to improve protein expression. For example, the expression of human recombinant enzyme $\mathrm{N}$-acetylgalactosamine-6-sulfatase (rhGALNS) in E. coli was undesirable due to protein aggregation. Several methods including the use of physiologically-regulated promoters, overexpression of native chaperones and applying osmotic shock were investigated to improve the production and activity of rhGALNS ${ }^{10}$. Protein activity, a phenotype representing the catalytic ability of a protein if it is an enzyme, is partly determined by its genotype (sequence of its coding gene). Directed evolution can effectively improve protein activity through changing the associated genotype, but this 
approach is resources-intensive. In the process of improving protein activity via directed evolution, mutagenesis is performed to change gene sequence and the mutated genes are inserted into plasmid used for transformation of a microbe, usually E. coli. Additional techniques are employed further to screen a large number of transformed cells for those that have higher protein activity. Since most of the protein directed evolution studies were only interested in the mutants with the highest activity, they did not reveal the genotype of most proteins that had lower activity. This fact has caused the challenge that almost no suitable database of protein activity is available for training computational models that can predict protein activity from protein sequence. Such models would greatly assist protein engineering by evaluating protein sequences in sil$i c o$. A suitable dataset for training the model should contain both protein activity data and the associated sequence data, and should be large enough $(>1,000$ entries).

Protein activity data cannot be easily pooled together for model training if they are related to enzymes that catalyze different chemistries, which is another reason why it

61 is difficult to generate the aforementioned datasets. The data of protein solubility from most types of proteins, however, can be compiled into one dataset, because protein solubility is a basic protein property. In this study and the relevant literature, protein solubility is defined as the percentage of a protein's soluble fraction ${ }^{11}$. It is a metric that is often used to assess the folding quality of a protein, under the assumption that incorrectly folded proteins form aggregates and are insoluble. Protein activity is thus correlated with protein solubility to some extent, because protein solubility may indicate the quality of protein folding which influences protein 3D structure and activity, i.e. proteins with higher solubility likely exhibit higher activity ${ }^{12}$. Improving the solubility of some recombinant proteins can enhance their production effectively ${ }^{13}$. Thus, protein

71 solubility may be used as a proxy for protein activity to develop predictive models that 
use protein sequence as input. With such a model, it would be possible to optimize the protein sequence of a protein in silico for improving its solubility and activity. For example, a Monte Carlo optimization method can be used as the procedures demonstrated in Figure 1: (1) a random change is introduced to the protein sequence, (2) the new protein sequence is evaluated by the model, and (3) if the predicted solubility is lower than that of the parental sequence, the change would be rejected, otherwise it would be accepted and used to initiate the subsequent iteration. This in silico optimization process may identify promising protein sequences to improve the success rate of the timeconsuming and labor-intensive experiments. If the protein activity heavily depends on its solubility, the experiment would identify new protein that has higher solubility and activity.

Machine learning has gained increasing attention recently in various fields, such as internet commerce, autonomous vehicles, and image recognition ${ }^{14,15,16,17, ~ 18, ~ 19, ~ 20, ~ 21, ~} 22$. Until now, a large number of machine learning methods have been explored to predict protein solubility from amino acid sequence ${ }^{6,11,23,24,25}$. Among the previous studies, we developed regression models that can predict protein solubility in the continuous values $^{26}$. Classification models which only label a protein as soluble or insoluble were developed in other studies but cannot be used in the in silico optimization, because it would mistakenly reject most changes that can result in a small but important increase in the protein solubility. So far, very few studies performed experimental validation of their solubility-prediction models and no study used such models to improve protein properties through the in silico optimization of protein sequence.

In our present study, based on a regression model that can predict protein solubility from protein sequence ${ }^{26}$, we developed optimization algorithms to increase predicted solubility under constraints that have been set after considering experimental feasibility 
and impact on protein function. The performance of the optimization process for improving protein solubility was validated successfully by experimentally measuring solubility. We found that adding short peptide rich in negatively charged amino acids was effective in improving solubility of many proteins. More importantly, we also verified

101 that activity of some proteins was indeed substantially improved when their solubility

102 was increased. Our study provides a generally effective approach to enhance protein solubility and activity.

\section{Results}

\section{Design the optimization methodology}

In order to improve protein solubility by in silico mutagenesis, we need to solve several

108 questions regarding how to change the protein sequence. One can change a protein sequence by adding amino acids to the sequence (addition), replacing amino acids in the sequence (mutation) and/or removing amino acids from the sequence (deletion). The

111 protein functions may be frequently abolished by mutation and deletion as the original

112 protein structure and active sites may be changed. To avoid such detrimental change to

113 the original function of the protein, addition was used in our study to change protein

114 sequence for improving protein solubility. The subsequent decision to make is how 115 many amino acids should be added. Adding too many amino acids would make exper-

116 imental validation to be more expensive and may also negatively affect the protein

117 function. Adding too few amino acids may not be able to improve protein solubility

118 substantially. We decided to evaluate adding 20 or 30 amino acids because adding more

119 than 30 amino acids to a protein by using synthetic oligonucleotides was experimentally 120 difficult. 
121 To optimize the sequence of the amino acids to be added, we designed an algorithm

122 based on the support-vector machine (SVM) prediction model we previously devel-

123 oped $^{26}$. The independent variable in the optimization function is the amino acid com-

124 position of the short peptide to be added, expressed as number of each amino acid in a

125 vector (Figure 1). The SVM model we developed only accepted amino acid composi-

126 tion of a protein as input, so we did not consider the full sequence information during

127 the optimization. Then the amino acid composition of a model protein with the added

128 amino acids was calculated and used as input for the SVM model. We used the genetic

129 algorithm (GA) which is a widely used algorithm for solving constrained optimization

130 problems. The objective function of GA outputs the predicted protein solubility by us-

131 ing the SVM model in the format of continuous values between $0-1$. The sum of the 132 number of amino acids added was set as 20 or 30 and the searching range for the number 133 of each amino acid added was from 0 to 20 or 30 .

\section{Optimize protein sequence in silico for improving protein solubility}

136 After designing this optimization algorithm, ten proteins with low solubility $(0.1)$ in the

137 eSol database (we had used the same database to train our machine learning model)

138 were selected as model proteins to test the algorithm (information of these proteins is

139 provided in Supplementary Table S2). The predicted solubility of all the ten proteins

140 was improved after adding 30 amino acids as peptide tags (Supplementary Figure S2).

141 One protein's solubility (name: agaW, N-acetylgalactosamine-specific enzyme IIC

142 component of PTS) was improved to 0.9951 from 0.1 after adding the designed short

143 peptide tags. When we allowed adding only 20 instead of 30 amino acids, the improve-

144 ment of predicted solubility slightly decreased (Supplementary Figure S2). Since it is 
145 easier and cheaper to add 20 amino acids in experiments than 30, we adopted adding

14620 amino acids as the constraint in the rest of this study.

147 To make this study more relevant to the imperative applications of recombinant en-

148 zymes, we selected six proteins which were important in engineering metabolic path-

149 way of E. coli to produce valuable metabolites (information of these proteins is pro-

150 vided in caption of Figure 2). These proteins' predicted solubility was lower than 0.6.

151 Adding 20 amino acids also substantially improved the predicted solubility of all the

152 six proteins (Figure 2). Three proteins (tal, $\mathrm{dxs}$ and valC) were chosen to experimentally

153 validate the optimization results since their original predicted solubility was low and

154 the predicted solubility was substantially improved through the optimization.

155 We also included agaw in the test because of the large improvement we observed in the

156 in silico optimization. The number of the amino acids to be added was allowed to be

157 decimal during the optimization and was rounded for experimental validation. The pre-

158 dicted solubility after rounding the number of the amino acids added was very similar

159 to that before rounding for all the tested proteins (Supplementary Table S6). To generate

160 sequence of the two tags to be added to a protein from the number of amino acids we

161 minimized the occurrence of amino acid repeats, which reduced the difficulty in syn-

162 thesizing the DNA. The sequence of the tags for those four proteins is listed in the

163 Supplementary Table S7.

165 Experimental validation of the optimized protein sequence

166 We constructed expression vectors to express the four proteins with and without the

167 optimized tags. Among them, protein agaw cannot be expressed (as determined by us-

168 ing SDS-PAGE) with and without the tags, which may be caused by the unstable protein 
structure or unsuitable experimental conditions. Protein valC can be expressed only without the peptide tags which may have impaired the protein stability. Protein tal and dxs were expressed with and without the tags (Figure 3). Protein solubility of tal and dxs was increased by $118 \%$ and $16 \%$ respectively by adding the tags.

By observing the amino acids added to dxs and tal (Figure $3 \mathrm{~b}$ and Supplementary table 5), it can be found that their peptide tags were dominated by aspartic acid (D) and glutamic acid (E). Aspartic acid and glutamic acid are the two negatively charged amino acids among the 20 amino acids. Adding them may introduce repulsive electrostatic interactions between protein molecules to prevent aggregation and to provide sufficient time for correct folding of proteins ${ }^{27}$. The similarity of the peptide tags inspired us to test whether one tag designed for one protein can be used to improve solubility of another protein. We found that the tags optimized for improving solubility of tal could also increase both predicted and measured solubility of dxs, and vice versa (Figure 4a). Another protein (name: ada, aldehyde dehydrogenase) used in a project of our laboratory was also tested with the tag designed for tal and its predicted and measured solubility were also enhanced (Figure $4 \mathrm{a}$ ). The results of switching tags suggested that the tags we designed may be generally effective in improving protein solubility.

\section{Protein activity also improved by the optimization}

The ultimate goal of this project was to improve activity of enzymes and their solubility was used as proxy because of the aforementioned reasons. Following the success of improving protein solubility, we measured activity of tal with and without the tags. Protein tal is tyrosine ammonia lyase which can deaminate tyrosine to produce couma- 
ric acid (Figure 4c). It is very useful in producing flavonoids by using engineered microbes $^{28,29}$. Tal activity was increased by $269 \%$ by adding the tags we designed for it (Figure $4 \mathrm{~d}$, based on $12 \mathrm{~h}$ reaction). The extent of the increase in activity was even larger than that in solubility, suggesting that adding the tags may also increase the expression level and/or specific activity of tal. This result proved that our optimization scheme for protein solubility was also effective for improving protein activity and using protein solubility as a proxy to increase protein activity was reasonable.

\section{Tags designed under more constrained conditions}

Among the four proteins selected for experimental validation, the protein valC (valencene synthase) cannot be synthesized only after the tags were added. This may be caused by the fact that the stability of protein valC was damaged after adding the tags. Our prediction model and optimization algorithm only took the protein solubility into account. However, other properties of the protein may be changed during the addition of highly charged tags, such as the protein stability. Therefore, we explored whether the peptide tags including mainly aspartic acid and glutamic acid can be replaced by tags that contain less charged amino acids to improve protein solubility.

The constrained condition that the number of aspartic acid and glutamic acid cannot be more than a threshold was therefore set in the optimization algorithm. The threshold was from 0 to 10 with step size of 1 for aspartic acid and glutamic acid respectively (Supplementary Table S8). When the limitation of addition number for aspartic acid and glutamic acid was reduced gradually from 10 , the predicted solubility was decreasing but the change was small. With the decrease in the number of aspartic acid and glutamic acid, the number of lysine $(\mathrm{K})$ increased substantially. Other amino acids only 
had a relatively small increase in the optimization solutions. When the constrained con-

217 dition was very strict, for example, no aspartic acid and glutamic acid were allowed,

218 the amino acids introduced were mostly alanine (A).

219 Another constrained condition was explored which limited the net charge of the peptide 220 tags. In this case, the upper bound for the absolute value of the net charge of the tag 221 was set as 5, 4, 3, 2, 1, and 0, respectively (Supplementary Table S9) and it could be 222 observed that the number of alanine increased most substantially with the decrease of 223 net charge, which was consistent with the results obtained under the other constraint 224 and supported that introducing alanine may be beneficial for the dissolution of protein 225 or the optimization failed to find a feasible solution under such stringent constraints.

226 This hypothesis was tested by doing experiments. The tags with net charge 1,3 , and 5 227 (Supplementary table S9) were used with protein valC. These new tags did not abolish 228 protein expression, confirming the hypothesis that excessive amount of aspartic acid 229 and/or glutamic acid may destabilize certain proteins. However, the solubility of protein 230 valC was not improved by the tags (Supplementary Figure S3). Protein valC may have 231 strong affinity to cellular membranes and thus cannot be solubilized by the designed 232 tags.

\section{Comparison with previous studies}

235 To improve protein solubility, some trial-and-error procedures were developed by in236 troducing small polyionic tags ${ }^{30,31,32}$. Small peptide tags have been used as solubility237 enhancing tags for a long time because they are short and less likely to interfere with 238 protein structure ${ }^{30}$. One study indicated non-polar surface and positively-charged 239 patches contributed to the separation of the soluble and insoluble proteins ${ }^{31}$. It was 
240 demonstrated that a concentration of positive charge may tend towards lower folded

241 state stability through unfavourable charge interactions and result in insolubility. In ad-

242 dition, a negatively charged fusion tag, NT11, was also developed to enhance protein

243 expression in E. coli ${ }^{32}$. However, these previous studies explored tags by trial and error

244 and cannot provide a generally useful quantitative model which can forecast perfor-

245 mance of tags with proteins which have not been tested. Among the diverse solubility-

246 enhancing tags that have been tested, the ones that are rich in aspartic acid and glutamic

247 acid were also studied before ${ }^{27}$.

248 To find out if the tags we obtained from our optimization were more effective than these

249 published ones, we compared them by using our predictive model and by conducting

250 experiments. We used tal as the model protein here, because its solubility was experi-

251 mentally confirmed to be low and its measured solubility can be substantially improved

252 by adding tags. The results were shown in Figure $4 \mathrm{~b}$ and protein tal without tag was

253 used as the control. All the three previously known polyionic tags increased solubility

254 of tal when added to tal, based on experimental measurement. But none of them out-

255 performed the tags identified in our optimization, supporting the usefulness of the tags

256 and the optimization procedure we reported here. In addition, there was a desirable

257 correlation between the predicted protein solubility and measured protein solubility.

258 The linear correlation between predicted solubility and measured solubility was quan-

259 tified by $\mathrm{R}^{2}$ with a value of 0.57 . Although the previous study exploded tags including

260 aspartic acid and glutamic acid by trial and error, our study provided better optimization

261 performance and a generally effective quantitative model. 


\section{Using machine learning for optimizing protein properties}

265 Using machine learning to assist the selection of proteins with specific properties has

266 been explored recently ${ }^{33,34,35}$. Heckmann et al. utilized machine learning to predict the

267 turnover number of enzymes in E. coli to optimize the growth rate, proteome composi268 tion and physiology of organisms ${ }^{34}$. And the prediction results were further used to pa269 rameterize two mechanistic genome-scale models more accurately. The machine learn270 ing model was trained by using the information of protein structure, biochemistry prop271 erties and assay conditions ${ }^{34}$, whereas protein sequences were used to train our predic272 tion model. Therefore, their model cannot be used to optimize protein sequence for 273 improving protein activity. Wu et al. incorporated machine learning into the directed 274 evolution workflow to help them identify proteins with high fitness value ${ }^{33}$. Then it was 275 applied to engineer an enzyme for stereodivergent carbon-silicon bond formation, a 276 new-to-nature chemical transformation. However, their training data for machine learn277 ing only included variants mutated at four amino acid residues. A protein might include 278 multiple positions for mutagenesis and information of four positions is not representa279 tive enough to train a machine learning model to handle other positions. The selection 280 of mutagenesis positions need to be customized by prior knowledge on the structure of 281 proteins. Yang et al. then reviewed the machine-learning-guided directed evolution fur282 ther $^{35}$. The different representation methods of protein sequence, prediction models, 283 optimization methods, and the training data of machine learning models were discussed 284 for different applications. Compared with the study mentioned above $\mathrm{e}^{33,35}$, we do not 285 need to train our optimization and prediction model again when we handle a new pro286 tein. In our study, we utilized the machine learning model to identify proteins with an287 other desired property, protein solubility. Our training dataset was obtained by using 
various proteins of E. coli and the optimization methodology did not need any customization and knowledge in biochemistry for new target proteins. With only the sequence information, our optimization model can provide effective guide for improving protein

291 solubility and activity. In addition, rather than using mutation to improve the protein 292 properties, we added small peptide tags to improve protein solubility and activity to avoid destroying the function of the original proteins.

\section{The contribution of aspartic acid and glutamic acid}

296 In this study, we designed a novel methodology to apply a predictive model of protein

297 solubility to improve protein solubility by adding short peptide tags. Aspartic acid and 298 glutamic acid dominated the tags that were obtained by using our optimization strategy.

299 This finding was consistent with the conclusion of an experiment we did to determine 300 which amino acids were the most important in determining accuracy of our solubility301 predicting model. In the experiment, we removed the percentage information of two 302 amino acids and evaluated the negative impact on the performance of the predictive 303 model. The model's inputs were composition of 20 amino acids, among which the per304 centages of 19 amino acid were independent. As a result, removing information of only 305 one amino acid would have no impact on model performance and we had to remove the 306 percentages of two amino acids. We evaluated all the combinations of two amino acids. 307 After removing aspartic acid or glutamic acid, the decrease of the prediction perfor308 mance represented by $\mathrm{R}^{2}$ was the most substantial (Figure 5), indicating they were the 309 most important ones for the model to be accurate. The causal relationship of the obser310 vations from this experiment and the optimization experiment could be that these two 311 negatively charged amino acids had large positive influence on protein solubility (as 
seen in the optimization experiment), so they were important to the accuracy of the model prediction (as observed in the importance analysis experiment). In addition, ar-

314 ginine which also showed some influence on the prediction performance when it was

315 removed, did not appear in the optimization results. This might be caused by that argi-

316 nine negatively affected the protein solubility and this hypothesis was tested (Supple-

317 mentary Figure S4). After adding 20 arginines to the six proteins from our laboratory,

318 all the predicted solubility was decreased. The suspected effects of glutamic acid, as-

319 partic acid and arginine were also supported by their spearman correlation coefficients

320 (Figure 5c), which were obtained by analyzing the large dataset we used to train our

321 model. There were some amino acids that were identified to be important by spearman

322 coefficient (Figure 5c) but were not found to be critical to model performance (Figure

323 5a), such as tryptophan and phenylalanine. It may be due to that spearman coefficient

324 alone is not sufficient to quantitatively describe the effects of amino acid on protein

325 solubility because of its qualitative nature and it did not consider abundance of other

326 amino acids (Figure 5b). In this study, we have shown that our machine learning model

327 is able to quantitatively describe the relationship and guide optimization of protein se-

328 quence.

329 When we trained the solubility-predicting model through machine learning, we did not

330 use any biochemistry knowledge. The optimization of protein tag to maximize protein

331 solubility was also purely mathematical without any dependence on prior knowledge.

332 Yet, the identified most beneficial amino acids and their influence on protein solubility

333 can be explained by using known biochemistry knowledge (electrostatic repulsion). As

334 to why the best tags were dominated by negatively charged amino acids rather than

335 positively charged ones, the reason might be that positively charged amino acids may

336 also improve protein solubility but their influence is less than those of negatively 
charged amino acids. When the number of the negatively charged amino acids was constrained, the optimization algorithm used positively charged amino acid (lysine) to improve protein solubility, which led to less improvement in solubility than using the negatively charged ones (Supplementary Table 8 and 9).

\section{Methods}

343 Protein database. All the information of protein solubility used in our study is from

344 the eSol database ${ }^{11}$ which is a unique database containing continuous values of protein 345 solubility. After removing items without sequence information according to the previ346 ous study ${ }^{26}, 3,148$ proteins in the eSol dataset were used for this study. In the study 347 which generated the dataset, the values of protein solubility were measured by synthe348 sizing the recombinant proteins by cell-free protein expression technology and then 349 being separated into soluble and insoluble fractions with centrifugation ${ }^{11}$. Solubility 350 was defined as the ratio of supernatant protein to total protein which was quantified by 351 SDS-PAGE.

353 Training flowsheet. The whole process of rationally engineering proteins with higher 354 solubility includes data pre-processing, training the SVM prediction model, construct355 ing an optimization methodology, and validating the methodology. As the first step, 356 amino acid composition was extracted from protein sequences by using Amino Acid 357 Composition Descriptor in protr package ${ }^{36}$ within R software, which converted charac358 ters of amino acids into numerical values indicating amino acid composition. For the 359 second part, the SVM model was built in MATLAB and trained following the same 360 procedure described in the previous study ${ }^{26}$. Then SVM was trained with the whole 
dataset to predict continuous values of protein solubility from amino acid composition.

362 For the third step, we filtered out a total of 58 proteins with low solubility of value 0.1

363 in the original dataset and 58 proteins were picked out. Proteins with long sequences

364 are more challenging to synthesize in experiments, therefore the protein sequences were

365 further filtered to have less than 333.3 amino acids $(1 \mathrm{~kb})$, which excluded 27 proteins

366 from the eSol database. Among the 27 proteins, the one with the minimum difference

367 between the predicted value and the real value of protein solubility, named glcE, was

368 selected as the sample protein to build a methodology for further optimizing protein

369 solubility. Genetic algorithm (GA), an optimization method, was explored to search for

370 maximum predicted solubility with constraints for the sample protein. The difference

371 between protein solubility before and after mutagenesis was used to evaluate the opti-

372 mization effect on protein solubility. Moreover, besides the sample protein, 10 proteins

373 with solubility of value 0.1 which have the least differences between predicted and

374 original solubility among the 27 proteins mentioned above were selected for applying

375 the optimization methodology. Six proteins commonly used in our laboratory were also

376 investigated for the optimization of protein solubility. Finally, among the 16 proteins

377 selected for optimization, 4 proteins that bear low solubility before adding the tags and

378 high predicted solubility after adding the tags were chosen for experimental validation.

379 The original and mutated protein sequences were synthesized to validate the change of 380 protein solubility by measuring the protein solubility with SDS-PAGE.

382 Machine learning models. The regression version of SVM used in this study could

383 also be named support vector regression (SVR) ${ }^{37}$. The aim of SVR is to solve ${ }^{38}$

$$
\operatorname{minimize} \frac{1}{2}\|w\|^{2}
$$




$$
\text { subject to }\left\{\begin{array}{l}
y_{i}-\left\langle w, x_{i}\right\rangle-b \leq \varepsilon \\
\left\langle w, x_{i}\right\rangle+b-y_{i} \leq \varepsilon
\end{array}\right.
$$

where $x_{i}$ is a training sample with target value $y_{i}$ and $w$ is the normal vector to the hy-

387 perplane. The inner product plus intercept $\left\langle w, x_{i}\right\rangle+b$ is the prediction value for that 388 sample. The difference of predicted values and true values for targets have to be within 389 an $\varepsilon$ range, which is a parameter serving as a threshold.

390 A regression machine learning model SVM in MATLAB was used for optimizing pro391 tein solubility for the all the proteins in our study and was validated by experiments 392 (Supplementary Table S10). The improved SVM model was used to optimize all the 393 proteins in silico and compared with the previous one in the Discussion.

395 Optimization algorithms. Genetic algorithm (GA), one of the evolutionary algo396 rithms, is inspired by the process of natural selection observed in nature ${ }^{39}$. It is a fre397 quently utilized randomized optimization algorithm for searching optima with con398 strained conditions. GA essentially simulates the way in which life evolves to find so399 lutions to real world problems. In GA, the solutions to a problem are represented as a 400 population of chromosomes evolving through successive generations. The offspring 401 chromosomes are generated by merging two parent chromosomes by crossover or mod402 ifying a chromosome by mutation. The offspring chromosomes are evaluated according 403 to the fitness or objective function in each generation. Chromosomes with higher fitness 404 values have higher possibility to survive and the process will stop when the offspring 405 chromosomes are almost identical or the terminal conditions set are reached. Strong 406 individuals will dominate the generation through many iterations in the process with 407 mutation, crossover and selection. The final chromosome represents an optimal or near408 optimal solution for the optimization problem. In our problem, the chromosomes are 
409 the sequence of peptide tags and the fitness function is the predicted solubility for pro-

410 teins after adding tags. Several hyperparameters can be tuned for the optimization al-

411 gorithm, such as the population size, the number of iterations for evolution and the

412 number of individuals mutating in each generation. We used a MATLAB Toolbox to

413 implement the optimization (iteration number $=1,000$, other parameters are provided

414 in Supplementary Table S1). The generic structure of GA in our study can be described

415 as follows:

begin:

initiate a tag representing by a 20 -dimensional vector with constrained conditions (sum of the vector is 20 and the value of each dimension is within range $0-20)$ evaluate the protein sequence after adding the tag;

while (if termination conditions are not met): do crossover and mutate parent tag sequences to yield offspring sequences;

evaluate the protein solubility for the proteins with offspring sequences; select and generate offspring sequence with higher solubility; end while; end.

429 Data visualization: The heat map was plotted by using the cmap function of the mat430 plotlib package in Python with the values of $\mathrm{R}^{2}$ after removing the information of two 
431 types of amino acids. The violin plot of the amino acid compositions was made by using

432 the violinplot function of the seaborn package in Python. Violin plot featured a kernel

433 density estimation of the underlying distribution. Spearman's rank correlation between

434 amino acid composition and solubility was computed using the spearmanr function of

435 the scipy.stats package in Python. The equation used was

$\rho_{\text {spearman }}=\frac{\sum_{i}\left(x_{i}-\bar{x}\right)\left(y_{i}-\bar{y}\right)}{\sqrt{\sum_{i}\left(x_{i}-\bar{x}\right)^{2} \sum_{i}\left(y_{i}-\bar{y}\right)^{2}}}$,

437 where the subscript $i$ denoted the ranks, and $x$ and $y$ represented amino acid composition 438 and solubility respectively.

440 Chemicals in experimental validation: All chemicals were purchased from Sigma441 Aldrich unless otherwise stated. All reagents used were of analytical grade. The DNA 442 oligomers used in this study were synthesized from Integrated DNA Technologies.

Plasmid construction: All the plasmids used in this work were constructed by using GT DNA standard ${ }^{40}$ (Supplementary Table S7).

Cell culture and SDS-PAGE analysis of protein solubility: Each of constructed plasmid was introduced into E. coli BL21 (DE3) (C2530H, New England Biolabs) for SDS-

449 PAGE analysis by using standard heat shock protocol. In order to test the resulting 450 strains, single colony was inoculated into $1 \mathrm{~mL}$ of $\mathrm{LB}$ with $100 \mu \mathrm{g} / \mathrm{mL}$ of ampicillin, 451 and was cultured overnight at $37^{\circ} \mathrm{C} / 250 \mathrm{rpm}$. Fifty microliters of the overnight grown 452 cell suspension were inoculated into $5 \mathrm{~mL}$ of $\mathrm{K} 3$ medium $^{40}$ with $100 \mu \mathrm{g} / \mathrm{mL}$ of ampi453 cillin. When cell was grown to $0.4-0.6$ optical density (OD) at 600 , isopropyl $\beta$-D-1- 
thiogalactopyranoside (IPTG) was added to a final concentration of $0.1 \mathrm{mM}$. After incubated overnight at $30{ }^{\circ} \mathrm{C} / 250 \mathrm{rpm}$, the cell culture broth was diluted to OD600 $=2.0$, and centrifuged at $5000 \mathrm{~g}, 10 \mathrm{~min}$. The obtained cell pellets were resuspended in 100 $\mu$ L B-PER II reagent (78248, Thermo Fisher Scientific). The mixtures were incubated for $15 \mathrm{~min}$ at room temperature with gentle rocking, and centrifuged at $16000 \mathrm{~g}$ for 20 min. The obtained supernatant contained soluble cell lysates. The insoluble cell pellets were resuspended in $100 \mu \mathrm{L}$ of $2 \%$ w/v SDS. Both soluble and insoluble cell pellets were analyzed by using SDS-PAGE (Mini-PROTEAN® TGX ${ }^{\text {TM }}$ Precast Protein Gels, 4561083, Bio-Rad). The image of the gel was processed and quantified by Gel Doc EZ Gel Documentation System (Bio-Rad).

Tal activity assay in vitro: One milliliter of obtained supernatant containing soluble cell lysates was added to $4 \mathrm{~mL}$ of PBS buffer ( $\mathrm{pH}=9.0)$ with $1 \mathrm{~g} / \mathrm{L}$ tyrosine (final concentration) in $50 \mathrm{~mL}$ falcon tube and incubated at $30{ }^{\circ} \mathrm{C} / 250 \mathrm{rpm}$. Three hundred microliters of samples were taken at $0 \mathrm{~h}, 1 \mathrm{~h}, 3 \mathrm{~h}$ and $12 \mathrm{~h}$ after incubation, and mixed with $700 \mu \mathrm{L}$ of acetonitrile to dissolve the produced $p$-courmaric acid (PCA). The mixture was incubated at $30^{\circ} \mathrm{C} / 250 \mathrm{rpm}$ for $1 \mathrm{~h}$, and then centrifuged at $13,500 \mathrm{~g}$ for $5 \mathrm{~min}$. Two microliters of the obtained supernatant was analyzed by using HPLC (Agilent 1260 Infinity HPLC) based on a previously reported method ${ }^{40}$.

\section{Supplementary information}

Supplementary data are available online. 


\section{Codes availability}

478 We present the optimization workflow as a series of notebooks hosted on GitHub 479 (https://github.com/xiaomizhou616/optimization_protein-solubility). The workflow 480 can be used as a template for analysis of other expression and solubility datasets.

\section{Reference}

1. Itakura $\mathrm{K}$, et al. Expression in Escherichia coli of a chemically synthesized gene for the hormone somatostatin. Science 198, 1056-1063 (1977).

2. Chan W-C, Liang P-H, Shih Y-P, Yang U-C, Lin W-C, Hsu C-N. Learning to predict expression efficacy of vectors in recombinant protein production. BMC Bioinform 11, S21 (2010).

3. Fang H, Li D, Kang J, Jiang P, Sun J, Zhang D. Metabolic engineering of Escherichia coli for de novo biosynthesis of vitamin B 12. Nature communications 9, 4917 (2018).

4. Lempp M, Farke N, Kuntz M, Freibert SA, Lill R, Link H. Systematic identification of metabolites controlling gene expression in E. coli. Nature communications 10, 1-9 (2019).

5. Esposito D, Chatterjee DK. Enhancement of soluble protein expression through the use of fusion tags. Current opinion in biotechnology 17, 353-358 (2006).

6. Idicula - Thomas S, Balaji PV. Understanding the relationship between the primary structure of proteins and its propensity to be soluble on overexpression in Escherichia coli. Protein Sci 14, 582-592 (2005).

7. Magnan $\mathrm{CN}$, Randall A, Baldi P. SOLpro: accurate sequence-based prediction of protein solubility. Bioinformatics 25, 2200-2207 (2009).

8. Trésaugues $L$, et al. Refolding strategies from inclusion bodies in a structural genomics project. Journal of structural and functional genomics 5, 195-204 (2004).

9. Ganesan A, et al. Structural hot spots for the solubility of globular proteins. Nature communications 7, 10816 (2016).

10. Reyes LH, Cardona C, Pimentel L, Rodríguez-López A, Alméciga-Díaz CJ. Improvement in the production of the human recombinant enzyme $\mathrm{N}$-acetylgalactosamine-6-sulfatase (rhGALNS) in Escherichia coli using synthetic biology approaches. Scientific reports 7, 5844 (2017).

11. Niwa T, et al. Bimodal protein solubility distribution revealed by an aggregation analysis of the entire ensemble of Escherichia coli proteins. Proc Natl Acad Sci USA 106, 4201-4206 (2009).

12. Zhou K, Zou R, Stephanopoulos G, Too H-P. Enhancing solubility of deoxyxylulose phosphate pathway enzymes for microbial isoprenoid production. Microbial cell factories 11, 148 (2012).

13. Kronqvist $\mathrm{N}$, et al. Efficient protein production inspired by how spiders make silk. Nature communications 8, 15504 (2017). 
14. Wu Y, et al. Google's neural machine translation system: Bridging the gap between human and machine translation. arXiv preprint arXiv:160908144, (2016).

15. Bojarski M, et al. End to end learning for self-driving cars. arXiv preprint arXiv:160407316, (2016).

16. LeCun Y, Bengio Y, Hinton G. Deep learning. nature 521, 436 (2015).

17. Silver $\mathrm{D}$, et al. Mastering the game of Go with deep neural networks and tree search. nature 529, 484 (2016).

18. Ferrucci D, Levas A, Bagchi S, Gondek D, Mueller E. Watson: Beyond Jeopardy! Artif Intell. (2013).

19. Godec $\mathrm{P}$, et al. Democratized image analytics by visual programming through integration of deep models and small-scale machine learning. Nature Communications 10, 1-7 (2019).

20. Weber T, Wiseman NA, Kock A. Global ocean methane emissions dominated by shallow coastal waters. Nature Communications 10, 1-10 (2019).

21. Li L, et al. Machine-learning reprogrammable metasurface imager. Nature communications 10, 1082 (2019).

22. Zador AM. A critique of pure learning and what artificial neural networks can learn from animal brains. Nature communications 10, 1-7 (2019).

23. Diaz AA, Tomba E, Lennarson R, Richard R, Bagajewicz MJ, Harrison RG. Prediction of protein solubility in Escherichia coli using logistic regression. Biotechnol Bioeng 105, 374-383 (2010).

24. Agostini F, Vendruscolo M, Tartaglia GG. Sequence-based prediction of protein solubility. $J \mathrm{Mol}$ Biol 421, 237-241 (2012).

25. Xiaohui $\mathrm{N}$, Feng $\mathrm{S}$, Xuehai $\mathrm{H}$, Jingbo $\mathrm{X}$, Nana L. Predicting the protein solubility by integrating chaos games representation and entropy in information theory. Expert Syst Appl 41, 16721679 (2014).

26. Han X, Wang X, Zhou K. Develop machine learning based regression predictive models for engineering protein solubility. Bioinformatics, (2019).

27. Paraskevopoulou V, Falcone F. Polyionic tags as enhancers of protein solubility in recombinant protein expression. Microorganisms 6, 47 (2018).

28. Jendresen $\mathrm{CB}$, et al. Highly active and specific tyrosine ammonia-lyases from diverse origins enable enhanced production of aromatic compounds in bacteria and Saccharomyces cerevisiae. Appl Environ Microbiol 81, 4458-4476 (2015).

29. Rodriguez A, Kildegaard KR, Li M, Borodina I, Nielsen J. Establishment of a yeast platform strain for production of p-coumaric acid through metabolic engineering of aromatic amino acid biosynthesis. Metabolic engineering 31, 181-188 (2015).

30. Bianchi E, Venturini S, Pessi A, Tramontano A, Sollazzo M. High level expression and rational mutagenesis of a designed protein, the minibody: from an insoluble to a soluble molecule. $J$ Mol Biol 236, 649-659 (1994).

31. Chan P, Curtis RA, Warwicker J. Soluble expression of proteins correlates with a lack of positively-charged surface. Scientific reports 3, 3333 (2013). 
32. Nguyen TKM, Ki MR, Son RG, Pack SP. The NT11, a novel fusion tag for enhancing protein expression in Escherichia coli. Applied microbiology and biotechnology, 1-12 (2019).

33. Wu Z, Kan SJ, Lewis RD, Wittmann BJ, Arnold FH. Machine learning-assisted directed protein evolution with combinatorial libraries. Proc Natl Acad Sci USA 116, 8852-8858 (2019).

34. Heckmann D, et al. Machine learning applied to enzyme turnover numbers reveals protein structural correlates and improves metabolic models. Nature communications 9, 5252 (2018).

35. Yang KK, Wu Z, Arnold FH. Machine-learning-guided directed evolution for protein engineering. Nature methods, 1 (2019).

36. Xiao N, Xu Q, Cao D. Protr: Protein sequence descriptor calculation and similarity computation with R. R package version 0.2-1. (2014).

37. Drucker H, Burges CJ, Kaufman L, Smola AJ, Vapnik V. Support vector regression machines. In: Advances in neural information processing systems (ed^(eds) (1997).

38. Smola AJ, Schölkopf B. A tutorial on support vector regression. Statistics and computing 14, 199-222 (2004).

39. Mitchell M. An introduction to genetic algorithms mit press. Cambridge, Massachusetts London, England, (1996).

40. Ma X, et al. A standard for near-scarless plasmid construction using reusable DNA parts. Nature communications 10, 3294 (2019).

\section{Acknowledgments}

We acknowledge the MOE Research Scholarship, MOE Tier-1 grant (R-279-000-452133) and NRF CRP grant (R-279-000-512-281) in Singapore. We thank Cortes-Pena

Yoel Rene for providing data visualization for data distribution and Spearman's rank

correlation tornado plot.

\section{Author contributions}

X.H. developed the optimization algorithms and statistical analyses. W.N. performed the experimental preparation and validation, and X.M designed and guided the experiments. All of them were supervised by X.W. and K.Z.. X.H. and W.N. wrote the manuscript with inputs from all the co-authors. All authors discussed the results and commented on the manuscript. 
bioRxiv preprint doi: https://doi.org/10.1101/817890; this version posted October 24,2019 . The copyright holder for this preprint (which

was not certified by peer review) is the author/funder. All rights reserved. No reuse allowed without permission.

621

622 Conflict of Interest: none declared.

623

624

625

626

627

628

629

630 
a

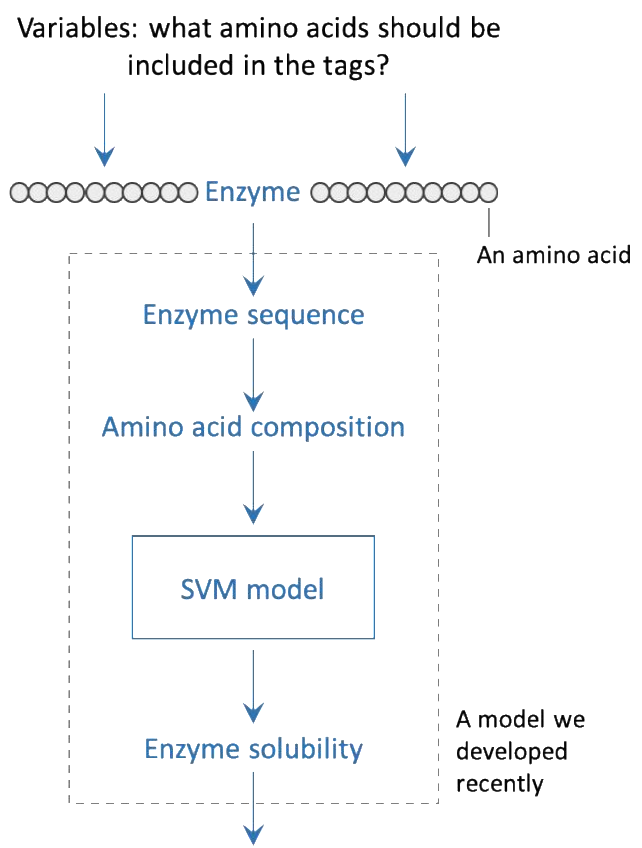

Objective: maximize enzyme solubility

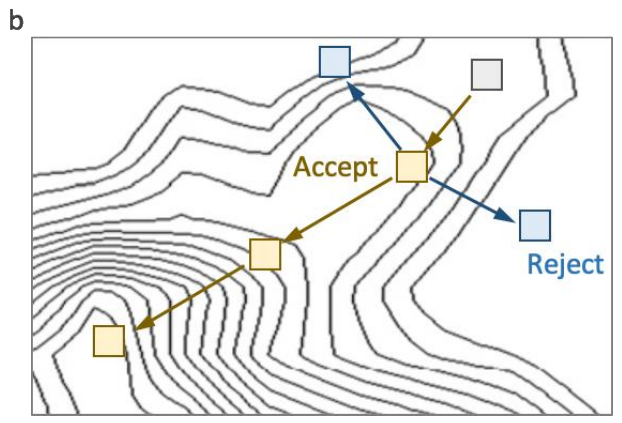

632 Figure 1 Machine learning model assisted optimization of protein solubility. (a) Illustration of the ob-

633 jective function when we aimed to improve protein solubility by adding short peptide tags. SVM: support

634 vector machine. A SVM regression model we recently developed was used in this study ${ }^{26}$. (b) Illustration

635 of the optimization algorithm. Genetic algorithm was used in this study. 


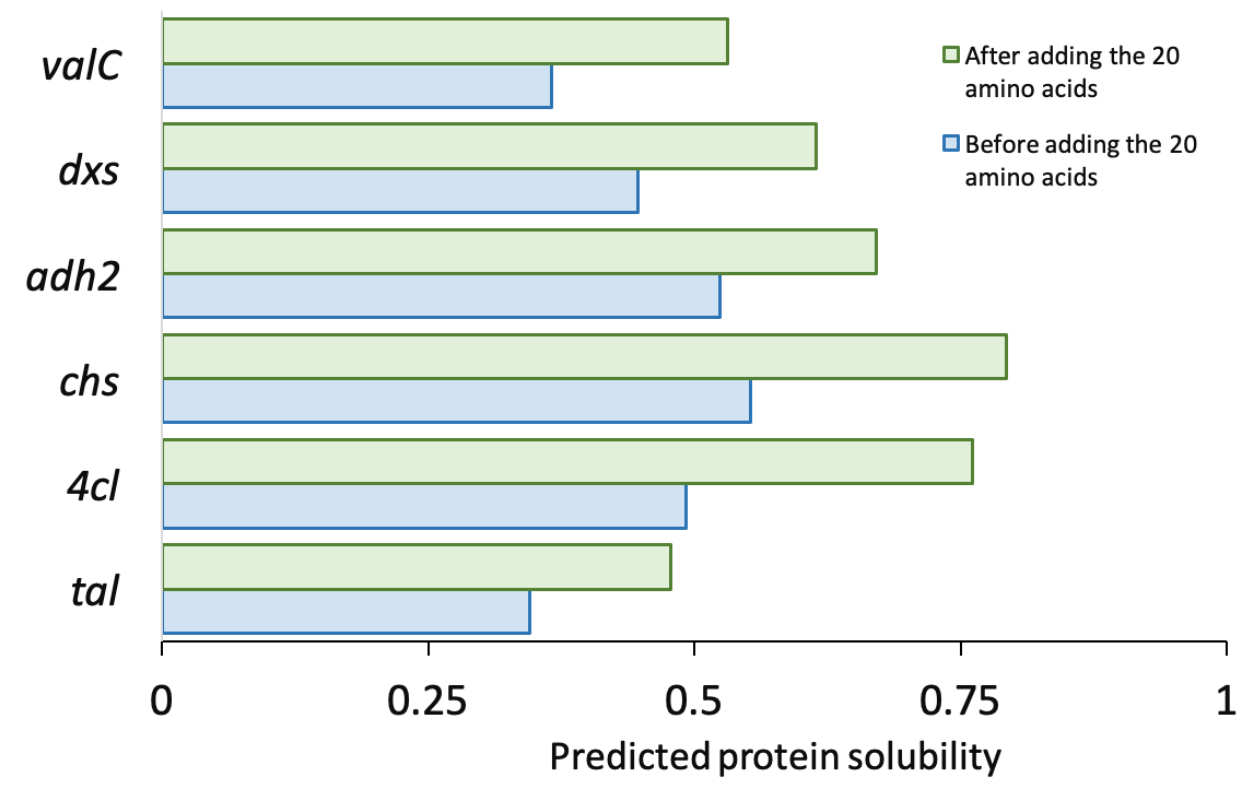

637 Figure 2 The predicted solubility before and after adding 20 amino acids for six proteins commonly used

638 by our laboratory. The six proteins were valC (valencene synthase), dxs (1-deoxy-D-xylulose-5-phos-

639 phate synthase), adh2 (alcohol dehydrogenase), chs (chalcone synthase), 4cl (4-coumarate-CoA ligase)

640 and tal (tyrosine ammonia-lyase). Their sequences were listed in Supplementary Table S7. Before adding

641 the tags, the protein solubility of them was predicted by SVM and recorded. Then GA was used to opti-

642 mize their solubility by adding 20 amino acids. The protein solubility after adding the tags was also

643 recorded for comparison.

644

645 Figure 3 (a) The SDS-PAGE analysis of protein tal and dxs expressed in E. coli with and without tags

646 designed by our optimization algorithm. "+” and "-” represented expressed proteins with and without

647 peptide tags respectively. "P" and "S" represented the pellet fraction (insoluble) and supernatant fraction

648 (soluble), respectively. The oval shapes highlight the bands of dxs and tal proteins. Protein tal and dxs a

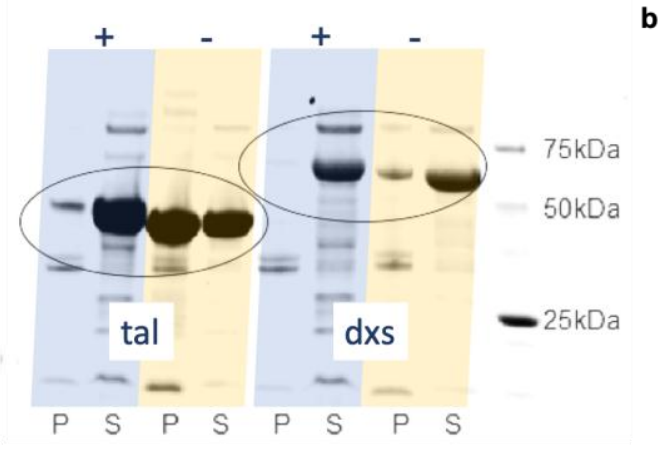

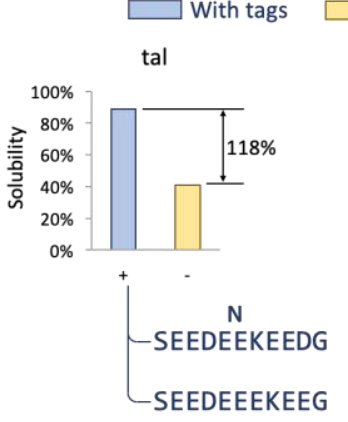

C

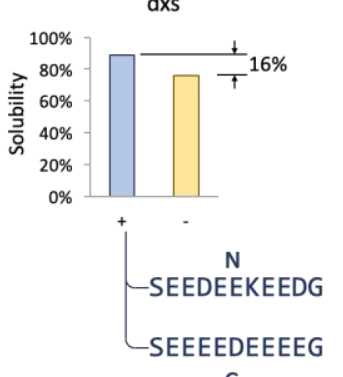

C 
650 PAGE images in a. The protein solubility was the ratio of soluble protein amount to the total protein

651 amount. The protein amount was estimated by using band intensity on SDS-PAGE images. The se652 quences of the designed tags for N-terminal and C-terminal were shown. The amino acid S and $\mathrm{G}$ on the 653 two ends of the tags were the linkers for GT DNA assembly standard, which was used to construct the 654 plasmids in this study ${ }^{40}$.

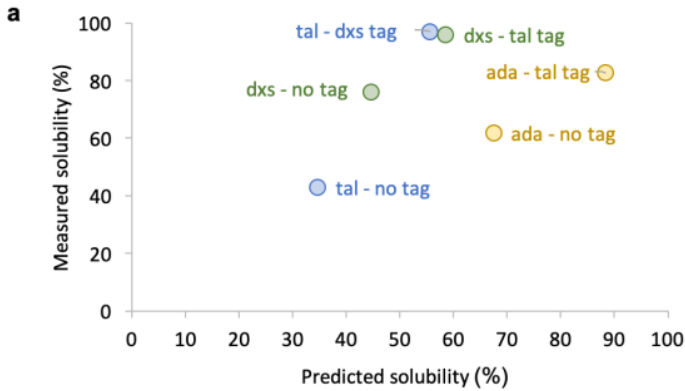

c

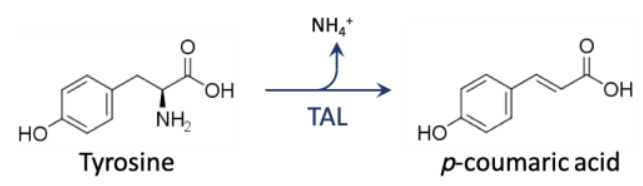

TAL: tyrosine ammonia lyase

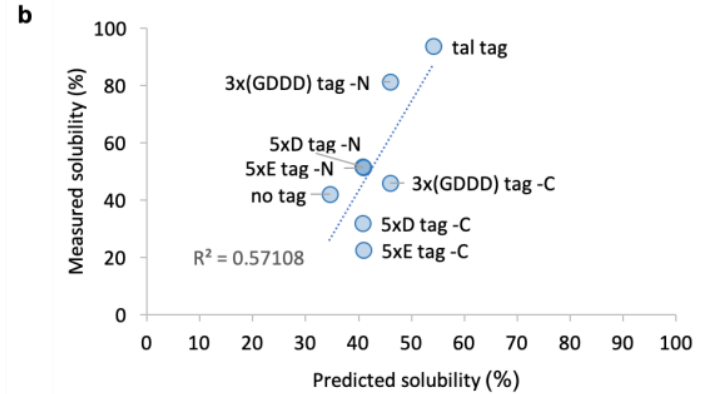

d

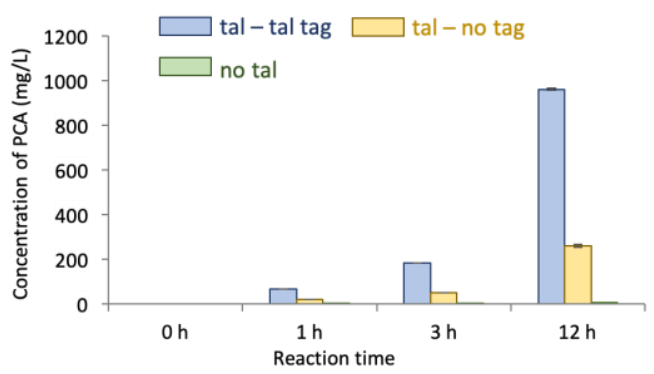

655

Figure 4 (a) The predicted and measured solubility of tal, dxs and ada after adding tags designed for other proteins. The purpose of switching tags for proteins was to test if the solubility-enhancing tags are generally effective in improving protein solubility. The same protein was labelled by using the same color to highlight the data before and after adding tags. In the data labels, the text before "-" indicates protein name and the text after "-" indicates the tags used if any. In the process of measuring the solubility, the protein expression condition was $\mathrm{K} 3$ medium with $20 \mathrm{~g} / \mathrm{L}$ glucose at $30{ }^{\circ} \mathrm{C}$. (b) The comparison of the tags designed in this study with tags used in previous studies. Protein tal was the only model protein used in this plot. No tag: solubility of tal without any tag. Tal tag: solubility of tal when we added the tags that were designed by our optimization algorithm for tal. 5xE tag -N/C: solubility of tal when $5 \mathrm{xE}$ tag (EEEEE) was added to its $\mathrm{N}$ - or $\mathrm{C}$-terminus. $5 \mathrm{xD}$ tag $-\mathrm{N} / \mathrm{C}$ : solubility of tal when $5 \mathrm{xD}$ tag (DDDDD) was added to its N- or C-terminus. 3x(GDDD) -N/C: solubility of tal when 3x(GDDD) tag (GDDDGDDDGDDD) was added to its $\mathrm{N}$ - or C-terminus. 5xD, 5xE and $3 \mathrm{x}(\mathrm{GDDD})$ were three tags used in a previous study and used here for comparison ${ }^{27}$. Since in previous study, only one tag was added 
669 to one protein, either at $\mathrm{N}$ - or C-terminus, we tested both cases for each tag. The two tags we designed

670 for tal were added to both ends of tal (Figure 1 and $3 \mathrm{~b}$ ). The sequences of all the tags are provided in

671 Supplementary Table S7. In the process of measuring the solubility, the protein expression condition was

$672 \mathrm{~K} 3$ medium with $20 \mathrm{~g} / \mathrm{L}$ glucose at $30^{\circ} \mathrm{C}$. (c) The reaction catalyzed by enzyme tal. (d) The protein

673 activity of protein tal before and after introducing tal tag. The product of the reaction catalyzed by en-

674 zyme tal was p-coumaric acid (PCA) and its concentration was used to indicate the activity of protein

675 tal. Cell lysate containing tal was used in the reaction. tal - tal tag: the strain containing tal with the tags

676 designed in this study. Tal - no tag: the strain containing tal without any tag. No tal: the strain that did

677 not express tal. The bars indicate the mean of six replicates. The error bars indicate standard error of six 678 replicates. 
a

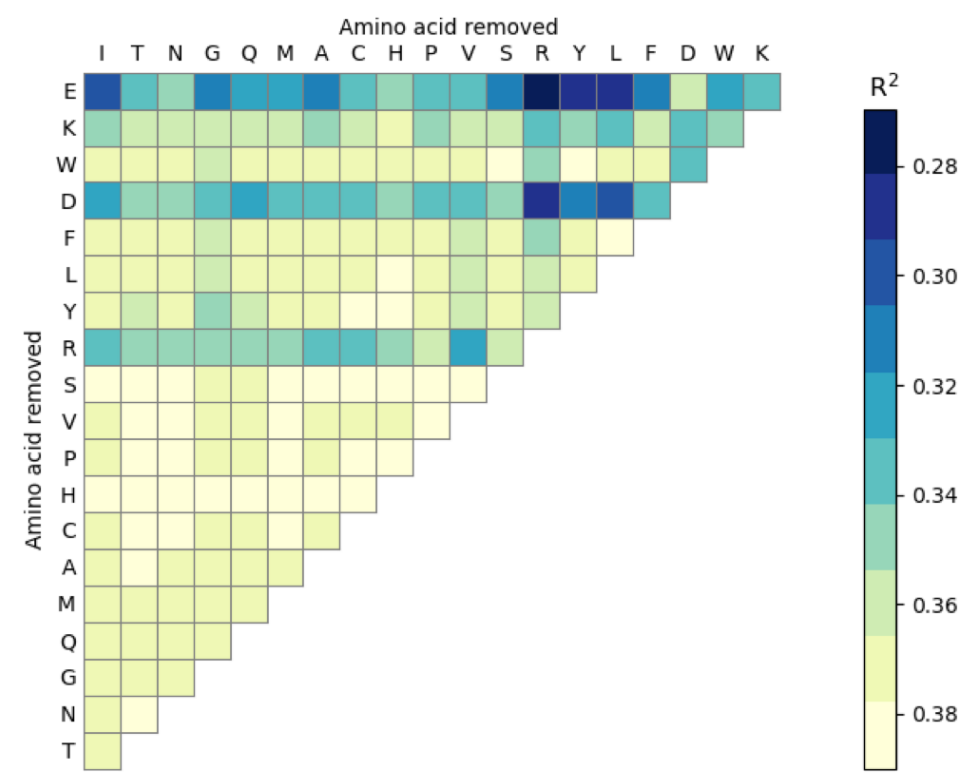

b

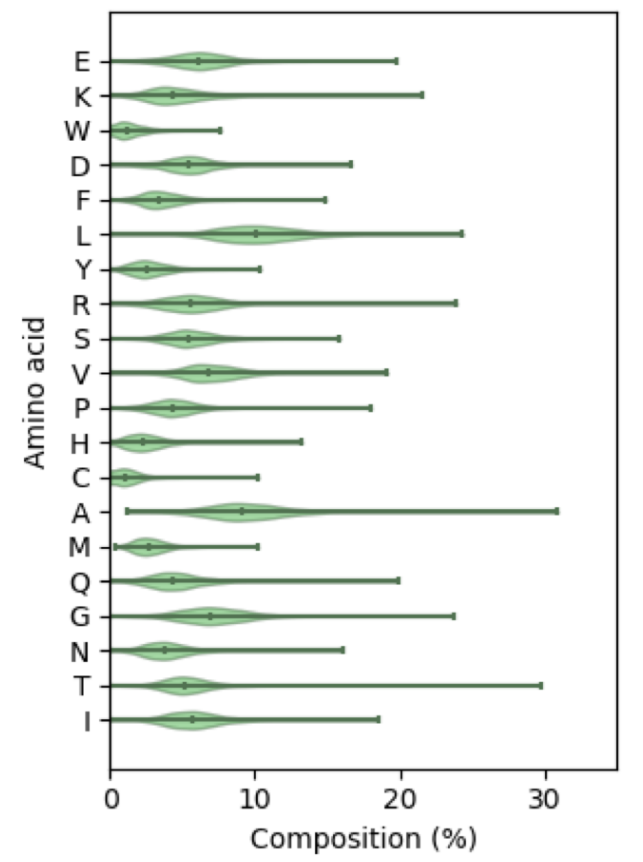

C

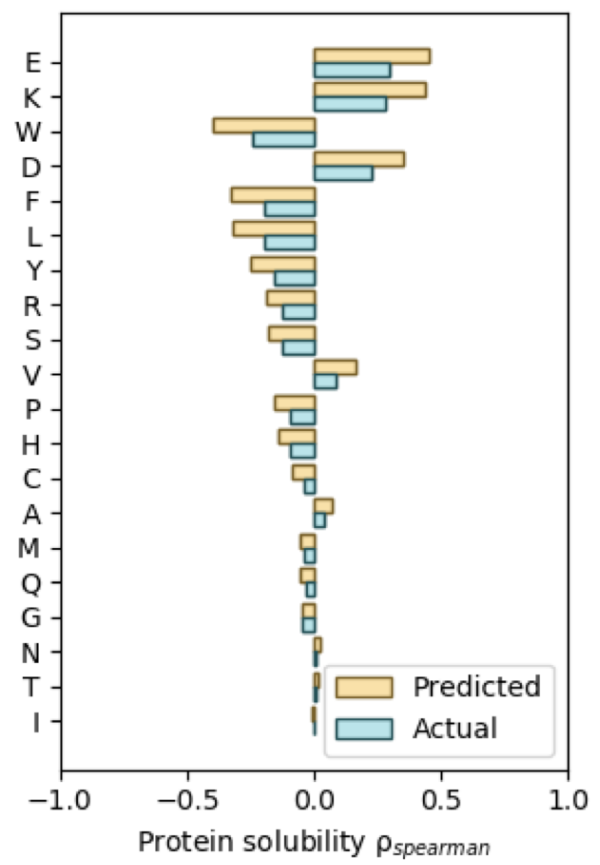

Figure 5 (a) Importance of various amino acids in determining the accuracy of the SVM regression model. The $\mathrm{R}^{2}$ of the SVM model was shown by using a heat map after removing the information of two

683 types of amino acids. Model training is described in Materials and Methods. Single letter amino acid

684 abbreviations are used in this figure. All the combinations of removing two types of amino acids are 685 tested and the performance of the resulting models is presented in the upper triangular matrix. Performance of the models was gauged by using $\mathrm{R}^{2}$, which is presented here by using color (a color bar is 
687 provided). The darker the color is, the more important the related amino acids are to the model perfor-

688 mance. (b) The distribution of amino acid composition (the input variables of the SVM model we used)

689 among all the proteins in the eSol database (the date source we used to train the SVM model). The violin

690 plot showed the mean value and the range of the amino acid composition used to train the SVM model.

691 (c) The Spearman's rank correlation between actual/predicted protein solubility and various amino acids.

692 Spearman's correlation, $\rho_{\text {spearman }}$, is a measure of monotonicity and represents the general sensitivity

693 of solubility to amino acid composition. A comparison between the Spearman's rank correlation tornado

694 plot for actual solubility and predicted solubility depicted how the model captured and magnified general

695 trends between amino acid composition and solubility. For example, for both the actual and predicted

696 solubility of proteins in the eSol dataset, the composition of D, E, or K was positively correlated with 697 solubility.

698 